\title{
Fabrication Methodology of microlenses for stereoscopic imagers using standard CMOS process
}

\author{
R. P. Rocha, J. P. Carmo, and J. H. Correia \\ Department of Industrial Electronics, University of Minho, Campus de Azurém, \\ 4800-058 Guimarães, PORTUGAL, \{rrocha,jcarmo,higino.correia\}@ dei.uminho.pt
}

This paper presents the fabrication technology of microlenses maintaining a high reproducibility of their characteristics with low cost. The objective of microlenses is to be integrated into imagers in CMOS technology to allow stereoscopic vision. The fabricated microlenses form cylindrical arrays to be placed above the optical filters and photodetectors, in order to potentiate stereoscopic vision and at the same time maximizing the color fidelity. An array of optical filters centered at the primary colors will enable a multicolor usage. The AZ4562 material was the photoresist selected for fabricating the microlenses. The cylindrical shape is obtained by reflowing the photoresist using the hot-plate technique.

\section{Introduction}

The available image sensors are not ready for stereoscopic acquisition. The stereoscopic vision as well as the high resolution enhances the quality of the images. The traditional solutions for acquiring tridimensional images are based on two or three monoscopic cameras, which must be perfectly synchronized with the penalty of losing the tridimensional effect or the emergence of artifacts in the images [1]. Moreover, it is very common the use of both depth sensors and monoscopic image sensors for doing the conversion between the bidimensional to the tridimensional domain [1].

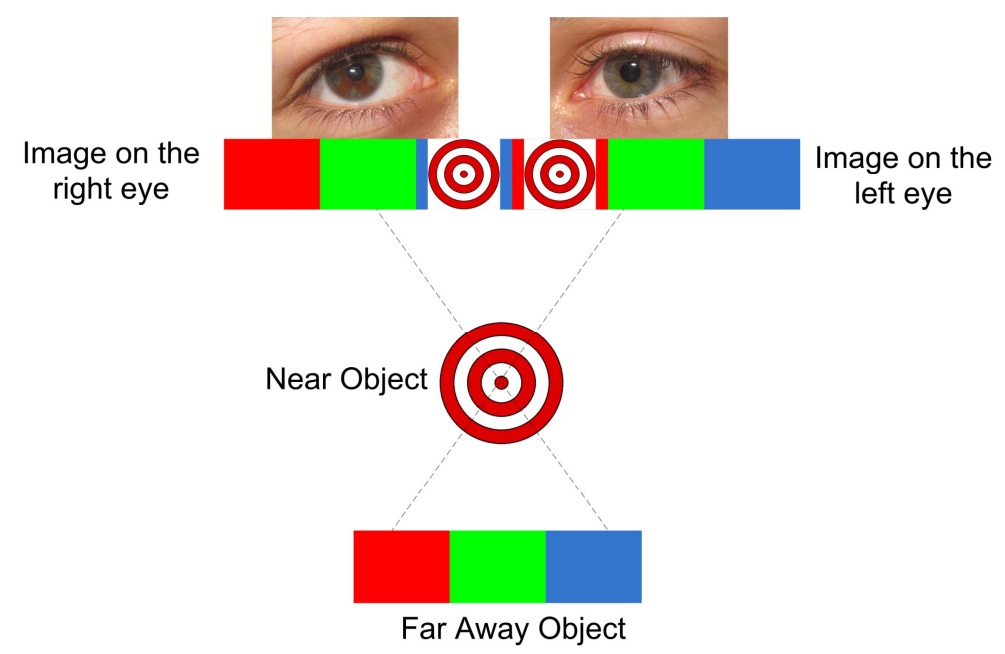

Figure 1. Illustration of the parallax effect for inducing sensation of depth in the brain [1].

The stereoscopic vision uses the parallax effect to cheat the brain for making it gain depth perception (which is also known as stereopsis), and thus the name "stereoscopy". This 
means that a stereoscopy with bad quality consequently induces perceptual ambiguity to the viewer [2]. This happens because the human brain is much more sensitive and less tolerant to bad stereoscopic images than to monocular images. Thus, a conscious of double vision can be caused by a multiplicity of factors, but all due to differences between the right and left images. The nature of such differences can be due to differences in brightness, differences in contrast, changes in reflection angle, differences in colors, and so on. Additionally, and according to the media experts, (even better) tridimensional image sequences can take few milliseconds to allow the brain and eyes to naturally adapt, to get the scene and adjust to it. The stereoscopic image sensor concept presented in this paper is composed by two entrance apertures (as it happens inside the human eyes) from where the left and right channels (the two images to be converted to the tridimensional domain) are passing before being focused by an objective lens into the sensitive area of the CMOS microdevice. The objective lens focuses the two incident beams (two viewpoints) in the direction of the microlens, where the light is concentrated in a small area (i.e., into the sensitive area of the CMOS photodiodes). After the passage by the optical filters, the individual rays of left and right viewpoints are steered towards the respective CMOS photodiodes. These two viewpoints are separated by focussing each side on the appropriate sensor column under the microlens and optical filters.

The fabrication of such a microdevice can be done using a cheap process because it allows the production of both the photodetectors and the read-out electronics within a reasonable cost. Finally, since the aforementioned process is very well characterized and known, the time to manufacture the first working prototype is expected to be short. This paper presents the design and fabrication technology of the microlenses for providing stereoscopic vision in a test-chip designed in the $0.7 \mu \mathrm{m}$ CMOS process from on-semiconductor foundry.

\section{Image sensor architecture and stereoscopic image formation}

As showed in the Figure 2, the image sensor is composed by two pupils (two entrance apertures just like the human eyes) from where the left and right channels (the two points of view that will originate the tridimensional effect) pass through before being focused by an objective lens. This lens focuses the two incident beams in the direction of the microlens, where the light is concentrated in a small sensitive area where the photodiodes are placed. After passing through the optical filters, the wavelengths coming from both the entrance apertures are directed towards the respective CMOS photodiodes. The two points of view are separated by focusing each side onto the appropriate sensor column under the microlens and optical filters.

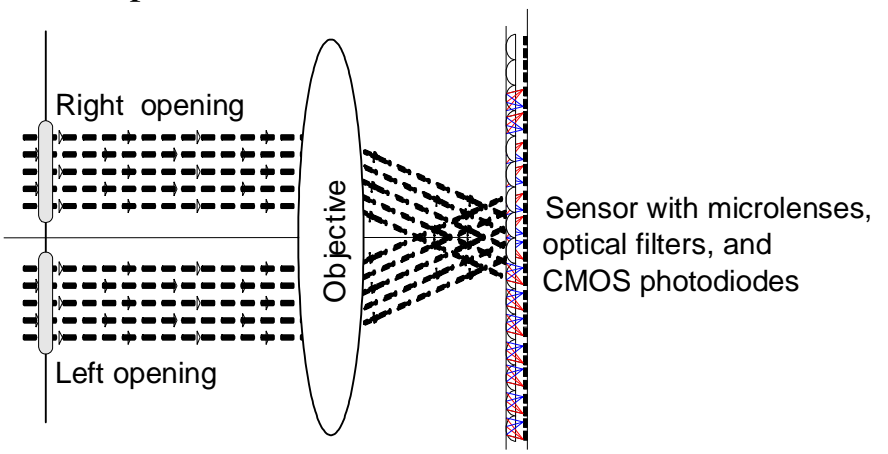

(a) 


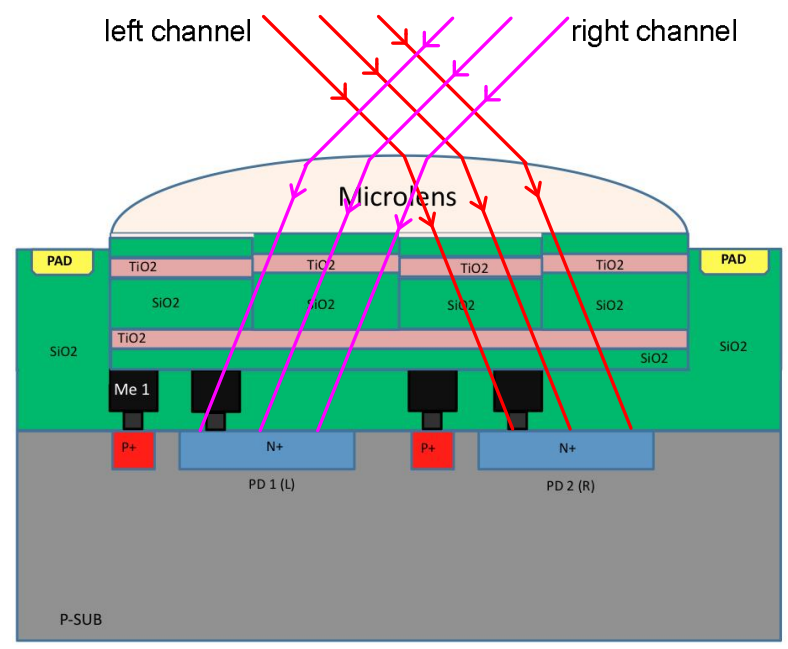

(b)

Figure 2. (a) Illustration of the stereoscopic image formation, and (b) illustration of the concept associated to the microlenses array for stereoscopic acquisition with a single polychromatic CMOS image sensor (example with one lens and two photodiodes).

Design

\section{Microlenses array}

There is available a huge number of materials for fabricating microlens such as the SU8/2, AZ9260 and AZ4562, for example. These polymers allow the microlens fabrication by thermally reflowing the raw material, whose processing steps of the reflow process are presented in the Figure 3. This permits the production of arrays containing a million or more microlens of good optical quality in just a few minutes and with high degree of reproducibility of their characteristics [3].
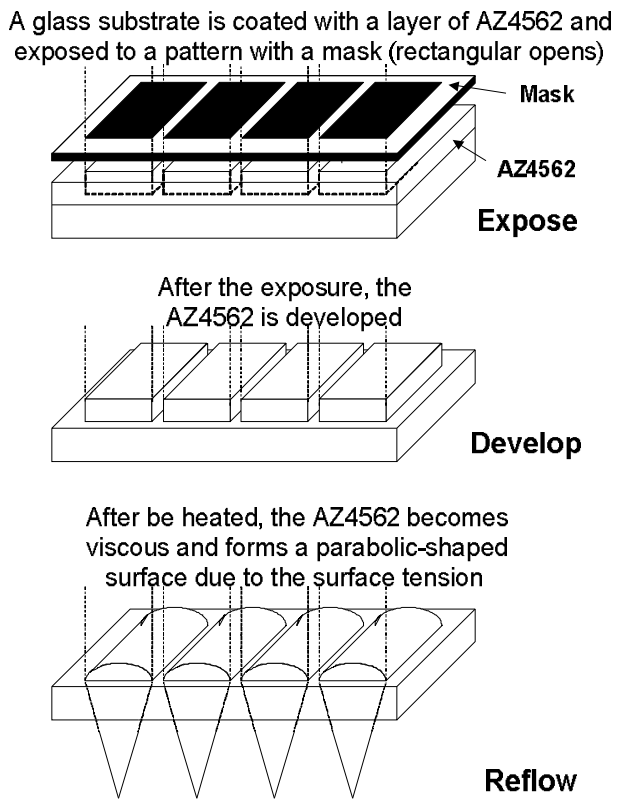

Figure 3. Microlenses array fabrication steps. 
There are few geometrical parameters to take in account before fabricating the microlenses. The Figure 4(a) shows the several geometrical parameters of a plano-convex lens: $n$ is the refractive index $n$ of the lens material (e.g., the AZ4562), $R[\mathrm{~m}]$ is the radius of a single spherical surface, $h[\mathrm{~m}]$ is the height of the surface undulation, $W[\mathrm{~m}]$ is the length of the transversal cut. The focal length $f[\mathrm{~m}]$ of the lens is straightforward to obtain: $f=(n-1) R$. Therefore, the major task is to obtain the radius $R$ in terms of this set of geometric parameters $\{h, W\}$. The next question that arises, is how to get $\{h, W\}$ in terms of the thickness $t h[\mathrm{~m}]$ of deposited AZ4562 as well as in terms of the resulted shape after doing the patterning. The Figure 4(b) shows the trapezoidal shape and geometric edges $\left\{W_{1}, W_{2}\right\}$ that is possible to obtain after the patterning task. The volumes (in this case, the transversal areas) of the material before $A_{1}=t h \times\left(W_{1}+W_{2}\right) / 2\left[\mathrm{~m}^{2}\right]$ and after $A_{2}\left[\mathrm{~m}^{2}\right]$ the reflow must be equal. $R$ is obtained keeping in account that $A_{1}=A_{2}$ where:

$$
A_{2}=\int_{-\frac{W}{2}}^{+\frac{W}{2}}\left(R^{2}-x^{2}\right)^{\frac{1}{2}} d x-W \cdot(R-h)
$$

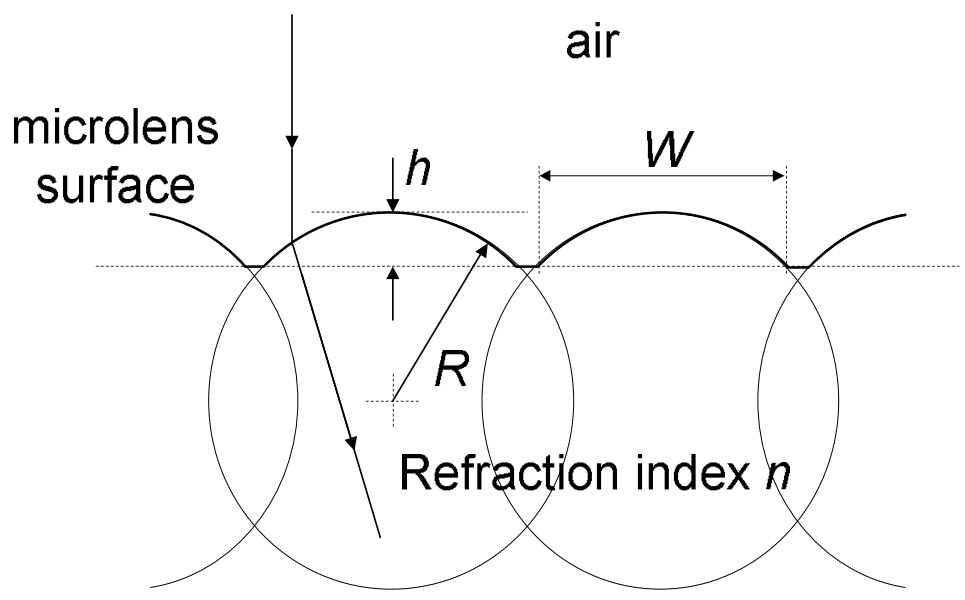

(a)

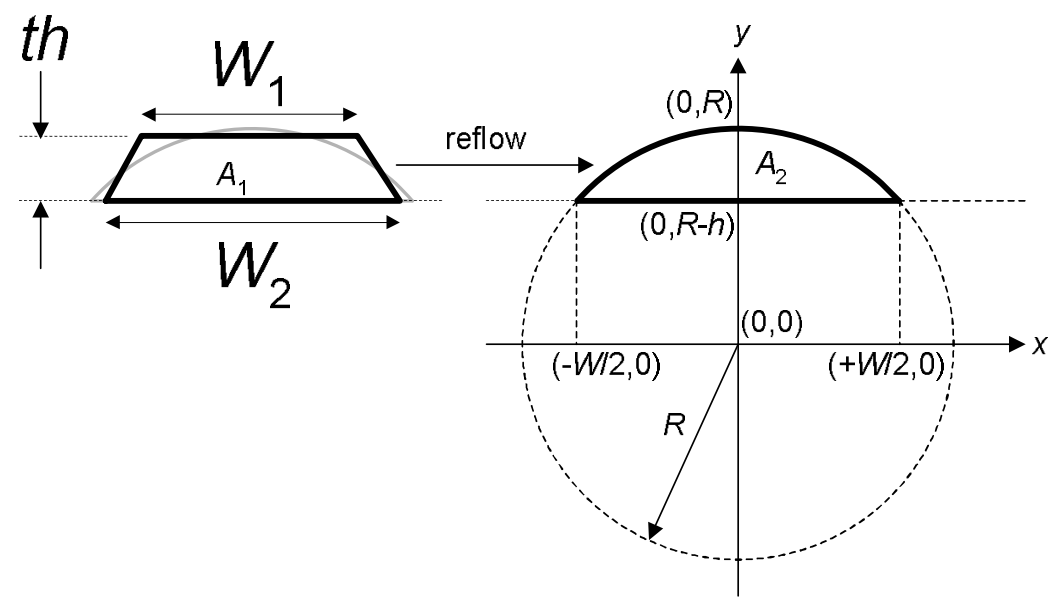

(b)

Figure 4. Factors determining the focal length of microlens and concept illustration. 
The Figure 5 shows (with the help of a selected sample) the concept to estimate the values of $R$, as well as the respective focal length $f$. For this selected sample $R \approx 21 \mu \mathrm{m}$, which combined with $n_{\mathrm{AZA562}} \approx 1.5936$ for $\lambda=580 \mathrm{~nm}$ (green) gives $f \approx 33.5 \mu \mathrm{m}$.

Good-quality lenses are fabricated with relatively crude lithography although greater consistency and reliability is achieved only if the process is carried out efficiently. The precise form of the microlens, and hence their focal properties are determined by the effects of surface tension. In particular the contact angle of the softened resist with the surface of the substrate will strongly influence the shape of the microlens.

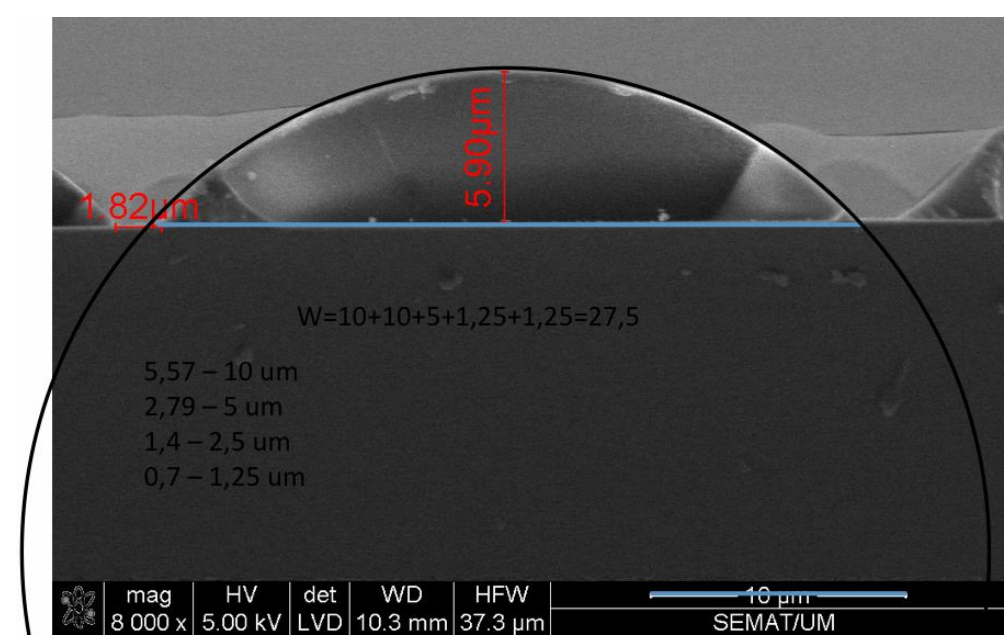

Figure 5. Factors determining the focal length of microlens and concept illustration.

\section{Fabrication}

The fabrication process illustrated in the Figure 3 allows the production of an array containing 142 microlenses. The rectangles that compose the mask array measure $4.9 \mathrm{~mm}$ in length, width of $30 \mu \mathrm{m}$ and $5 \mu \mathrm{m}$ spacing between adjacent rectangles. This setup allows the fabrication of good optical quality in just a few minutes and with high degree of reproducibility of their characteristics. To the author's knowledge, this is the first time that such a geometry with the presented dimensions and aspect ratio, is used to fabricate an array of microlenses. Different sized arrays were de-signed and printed into a $128 \mathrm{kdpi}$ mask with each array covering an area of $5 \mathrm{~mm}^{2}$. The rectangles are coated with chromium making them opaque to light and the spacing between rectangles is transparent allowing the photoresist under it to be exposed to the UV light later on. The fabrication process of the microlenses array requires several steps and process parameters summarized in Table 1. First, it is necessary to spin coat the AZ4562 at $6000 \mathrm{rpm}$ during 20 seconds, in a previously cleaned substrate, to achieve the desired $5 \mu \mathrm{m}$ thickness.

TABLE I. Fabrication Process steps and parameters.

\begin{tabular}{ll}
\hline Process steps & Process parameters \\
\hline Spin coating & 20 seconds @ 6000 RPM \\
\hline Prebake (hotplate) & 5 minutes @ $100{ }^{\circ} \mathrm{C}$ \\
\hline Exposure (mask aligner) & 30 seconds in contact mode @ $134 \mathrm{~W}$ \\
\hline Developing & AZ400K or AZ351B developers in a 1:4 concentration with distilled water \\
& $(2 \times 2$ minutes and 15 seconds $)$ \\
\hline Cleaning & Rinse with distilled water and dry with $\mathrm{N}_{2}$ flow \\
\hline Thermal Reflow (hotplate) & 5 minutes @ $130^{\circ} \mathrm{C}$ \\
\hline
\end{tabular}


After the coating, a prebake phase, using a computer controlled hot-plate at $100{ }^{\circ} \mathrm{C}$ for 5 minutes, is necessary to evaporate the solvents present in the photoresist. Next, to obtain the required array-like structure, the mask with the correspondent geometry is placed on top, directly contacting with the coated photoresist and exposed to UV light using a mask aligner. The AZ being under a $134 \mathrm{~W}$ exposure during 30 seconds makes the unexposed material insoluble. Afterwards, the developing phase is achieved by either the AZ400K or the AZ351B developers in a 1:4 concentration with distilled water. To accomplish it, the substrate is immersed into two developer baths for 2 minutes and 15 seconds each, in a magnetic stirrer plate. This is required to leave just the unexposed photoresist in the substrate. The photolithographic process ends with the parallelepiped structures being rinsed with distilled water and dried out with a nitrogen flow. Finally, to obtain the lens profiles, the thermal reflow technique is applied so the substrate with the array containing the fabricated structures is placed on a hotplate at $130{ }^{\circ} \mathrm{C}$ for 5 minutes.

\section{Results}

The Figures 6 and 7 show two photographs of the photoresists array before and after the reflow step, respectively. The parts (a) and (b) of correspond to a single element of the array and an overview of the array, respectively. These structures refer a sample prototype built using photolithography with a chromium-on-glass mask. It is clear from the previous figures that the thermal reflow process permitted obtaining the desired microlens profile to concentrate the light into specific directions.

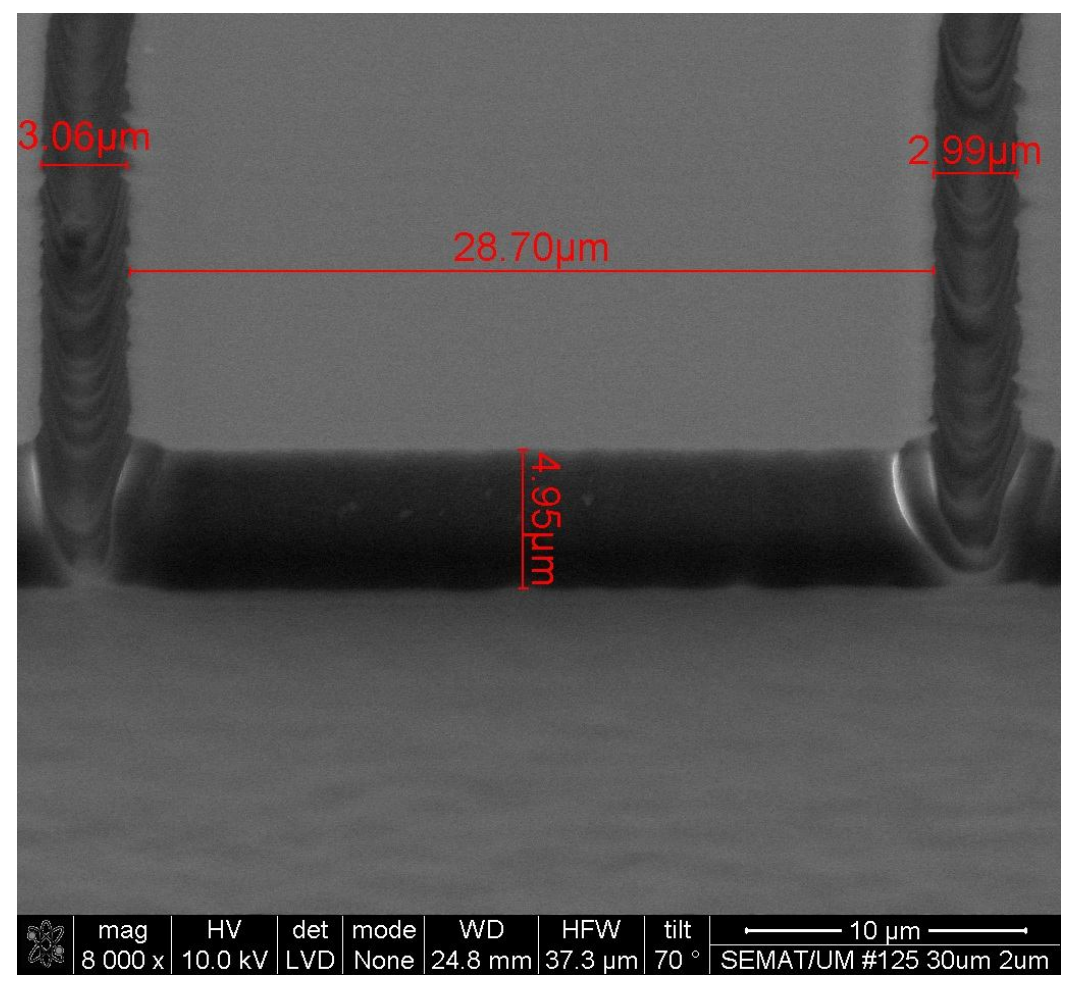

(a) 


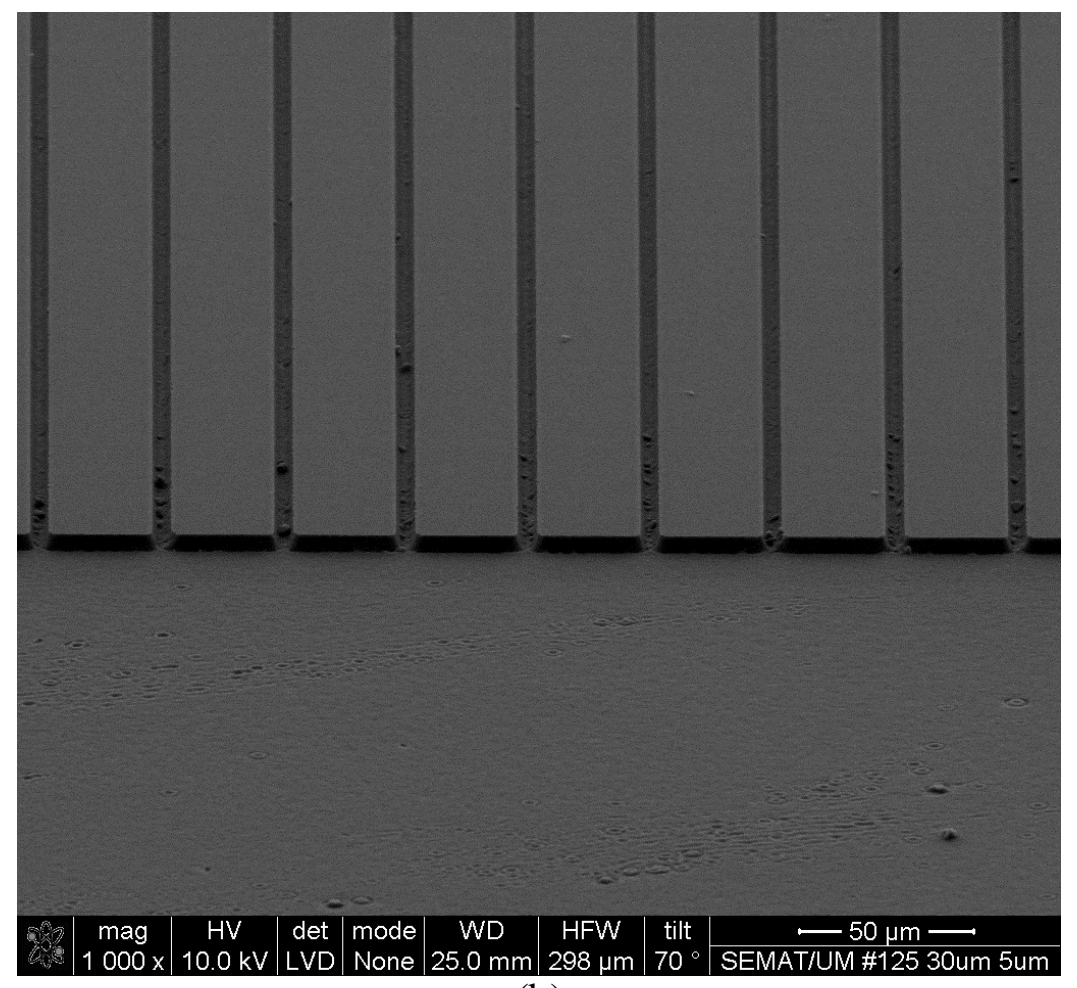

(b)

Figure 6. SEM images (a) of a single element and (b) an overview of the array. These photographs were taken from an array of microlenses before doing the thermal reflow.

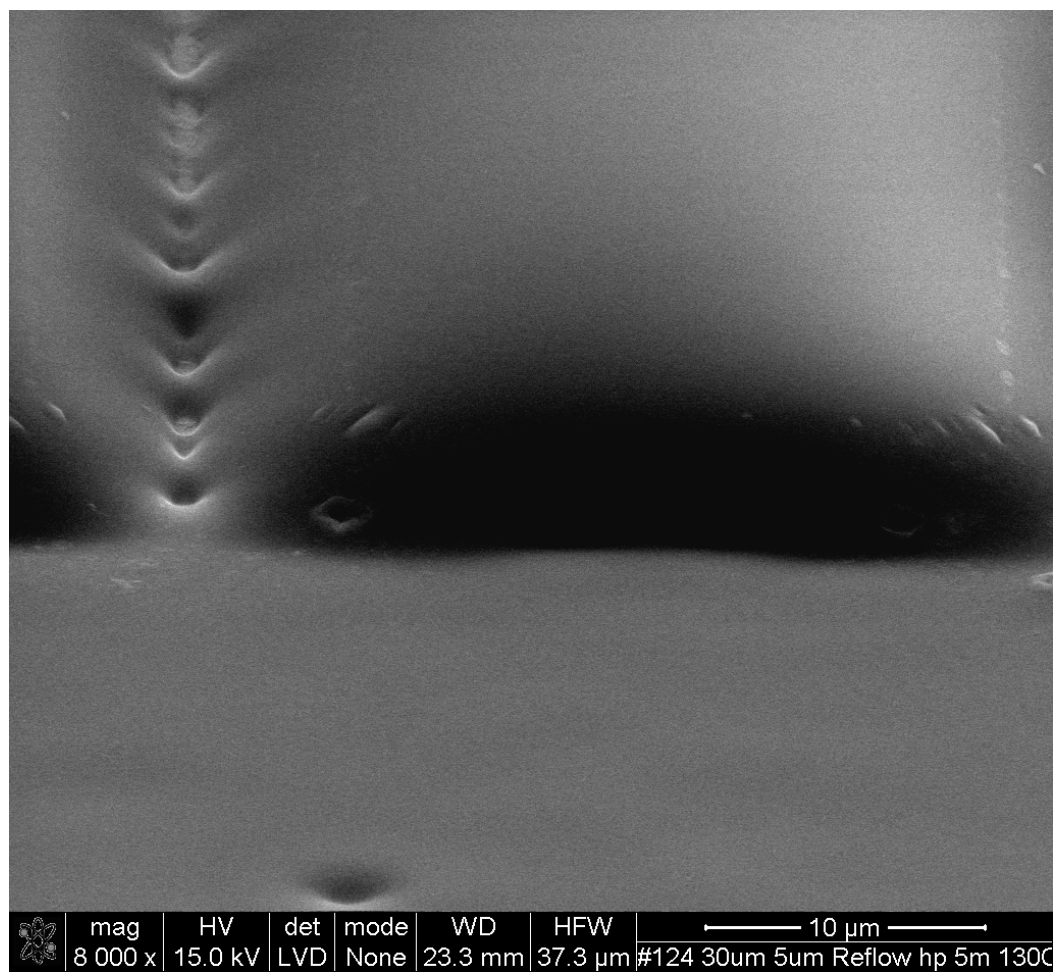

(a) 


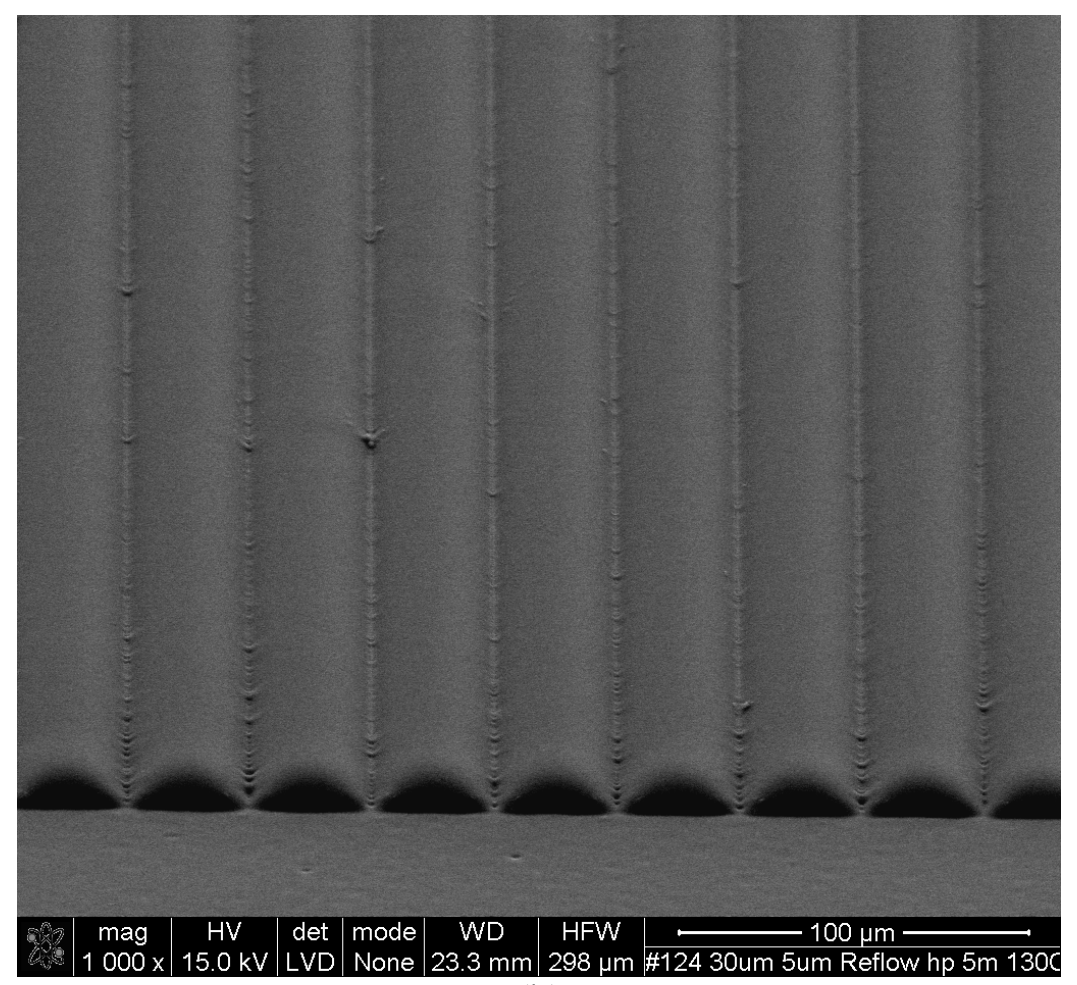

(b)

Figure 7. SEM images (a) of a single element and (b) an overview of the array. These photographs were taken from an array of microlenses after doing the thermal reflow.

\section{Conclusions}

This paper presented a microlenses fabrication process for integration on a stereoscopic image sensor in CMOS technology for use in biomedical devices. The microlenses design started with the FEM simulations to set some parameters needed to fulfill the desired objectives. Each photodetector measures a specific wavelength with is diffracted by the microlenses. The complete fabrication process was explained and the initial and final structures obtained were physically characterized. It was shown that the reflow step is what determines the actual microlenses profile. The several steps that comprise the photolithographic fabrication process were done with a chromium-on-glass mask and a mask aligner as the UV light source.

\section{Acknowledgments}

This work and Rui Pedro Rocha were fully sup-ported by the Portuguese Foundation for Science and Technology under the project FCT/PTDC/EEA-ELC/109936/2009 and the financial grant SFRH/BD/33733/2009, respectively. The authors also acknowledge to Dr.-Ing Christian Koch from MicroChemicals GmbH for the technical support.

\section{References}

1. I. Andorko, P. Corcoran, P. Bigioi, in Proceedings of OPTIM 2010, Brasov, Romania, 20-22 May 2010, 920-925.

2. S. Zeki, Consciousness and Cognition, 13, 173-196.

3. H. Takahashi, et al, IEEE Journal of Solid State Circuits, 39(12), 2417-2424. 